\title{
Matching and nonmatching concept learning in rats
}

\author{
ESHO NAKAGAWA \\ Kagawa University, Kagawa, Japan
}

\begin{abstract}
In three experiments, rats' ability to form a relational concept was examined. In Experiment 1, they were trained on a matching task under a 2-sec delay, a 0-sec delay, or a simultaneous condition. Group 2-sec delay learned the nonmatching as rapidly as the other groups. In Experiment 2, rats were trained on a delayed matching or nonmatching task and then under either shift or nonshift conditions by using stimuli that recurred over trials within each session. Performance on the first trial in Phase 2 between Groups Nonshift (75\% correct) and Shift (25\% correct) have been symmetrically displaced from chance level. In Experiment 3, rats trained on a same-different task in Phase 1 learned a shift task more rapidly than those trained on a discrimination task in Phase 1. These results produced several converging lines of evidence that suggest that rats have the ability to learn the abstract concepts of matching-nonmatching and same-different.
\end{abstract}

Zentall and Hogan (1974) trained some pigeons on matching and others on oddity with one pair of stimuli, and then transferred them to a new pair of stimuli, either with same rule holding as in the first problem (i.e., matching if the first problem was matching or oddity if the first problem was oddity), or with the opposite. Pigeons that had not shifted to the opposite task performed better than those that did. That is, the nonshifted pigeons learned the shift task more rapidly than the shifted pigeons did. This effect was replicated with a similar shifted versus nonshifted design for a go/no-go version of the matching and oddity task (Zentall \& Hogan, 1975) and in a conventional matching-to-sample experiment using transfer to colors after overtraining on geometric patterns (Zentall \& Hogan, 1976). Zentall and Hogan have interpreted the results of these experiments as demonstrating that pigeons can learn a relational concept. Further evidence appearing to support this claim has been reported by Lombardi, Fachinelli, and Delius (1984), Urcuioli and Nevin (1975), and Urcuioli (1977).

Few studies have reported that rats have the ability to form a relational rule. Lashley (1938a, 1938b) has reported that rats cannot learn an oddity problem, but Wodinsky and Bitterman (1953) have suggested that rats do have this ability.

Aggleton (1985), Mumby, Pinel, and Wood (1990), and Rothblat and Hayes (1987) have shown that rats can generalize matching and nonmatching rules across trial-unique stimuli. When trial-unique (nonrecurring) stimuli are used, rats are likely to solve the nonmatching-to-sample task using stimulus-recognition memory.

Nakagawa (1992) has shown that rats can acquire and transfer a matching or nonmatching concept across pairs

Requests for reprints should be sent to E. Nakagawa, Department of Psychology, Kagawa University, 1-1 Saiwai-Cho, Takamatsu, Kagawa, 760 , Japan. of stimuli using a very restricted set of repeated stimuli in simultaneous matching and nonmatching learning situations-the sample is presented on the center entrance and the two comparisons are presented on the side entrances, one being identical to the sample. In such learning situations, rats can learn matching and nonmatching tasks on the basis of response tendencies, such as selecting the larger door or the smaller door, rather than the same or different door.

One way to demonstrate that rats can learn a relational concept is the delayed matching or nonmatching procedure, in which the comparison stimuli are turned on immediately as the sample is turned off.

Aggleton (1985) has suggested that it is difficult for rats to acquire and transfer delayed matching or nonmatching when using a very restricted set of repeated stimuli.

The purpose of the present study was to examine whether rats could learn the relational concept of same or different. In order to determine this, first it was necessary to confirm that they could acquire matching or nonmatching learning by using stimuli that recur over trials within each session in the delayed condition. Next, it was necessary to investigate the transfer of a matching or nonmatching concept across orthogonal dimensions. In Experiment 1 , it was examined whether rats could acquire nonmatching in delayed conditions (2-sec and 0 -sec) as well as in a simultaneous condition by using stimuli that recurred over trials within each session. If it is difficult for rats to acquire and transfer delayed matching or nonmatching learning by using a very restricted set of repeated stimuli, as suggested by Aggleton (1985), then they should learn a nonmatching task in the delayed condition more slowly than in the simultaneous condition, or they should not be able to learn a nonmatching task in the delayed condition. By contrast, no significant between-group differences in the speed of learning would indicate that rats can learn a delayed nonmatching task by using a very re- 
stricted set of repeated stimuli. The issue of transfer of matching or nonmatching was examined in both the $2-\mathrm{sec}$ delayed matching or nonmatching procedure (Experiment 2) and the same-different task (Experiment 3).

\section{EXPERIMENT 1}

\section{Method}

\section{Subjects}

Twenty-four experimentally naive female Sprague-Dawley rats were used. They were about 200 days old with an initial average body weight of $260 \mathrm{~g}$. The animals were handled for $5 \mathrm{~min}$ a day for 12 days, and were maintained on a daily 2 -h feeding schedule prior to the experiment. The amount of food in the daily ration was gradually reduced until the body weight of each animal reached $80 \%$ of the baseline weight at the start of the experiment. Water was always available for the animals in their individual home cages. The animals were maintained on a 7:17-h light:dark cycle, with lights off at 7:00 p.m.

\section{Apparatus}

An automatic T-maze (Toyo Physical Ltd), shown in Figures 1 and 2, was used. The apparatus was painted medium-gray inside, and was illuminated throughout the experiment by a 10 -W fluorescent lamp suspended horizontally $40 \mathrm{~cm}$ above the top of a choice chamber. The apparatus consisted of a runway $(30 \mathrm{~cm}$ in height, $12 \mathrm{~cm}$ in width, and $25 \mathrm{~cm}$ in length) with a startbox $(30 \mathrm{~cm}$ in height, $12 \mathrm{~cm}$ in width, and $15 \mathrm{~cm}$ in length) and a choice chamber $(30 \mathrm{~cm}$ in height, $56 \mathrm{~cm}$ in width, and $32 \mathrm{~cm}$ in length). The

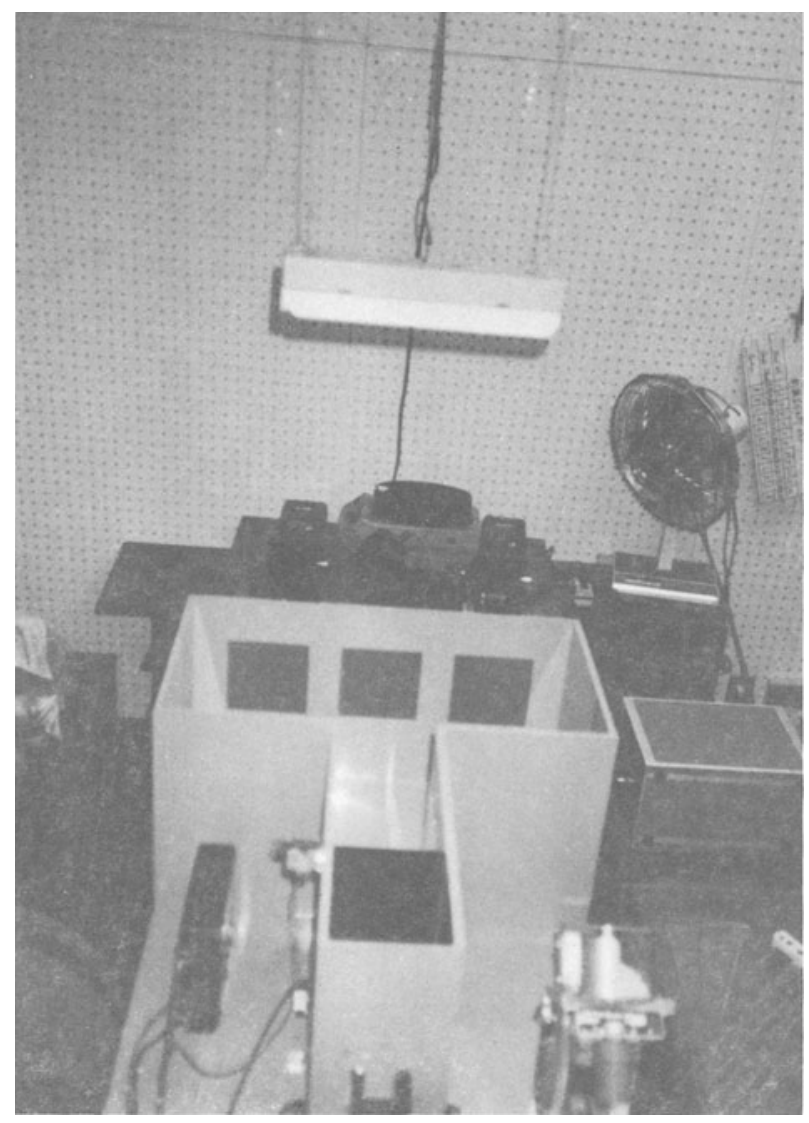

Figure 1. The automatic T-maze used in Experiments 1, 2, and 3.

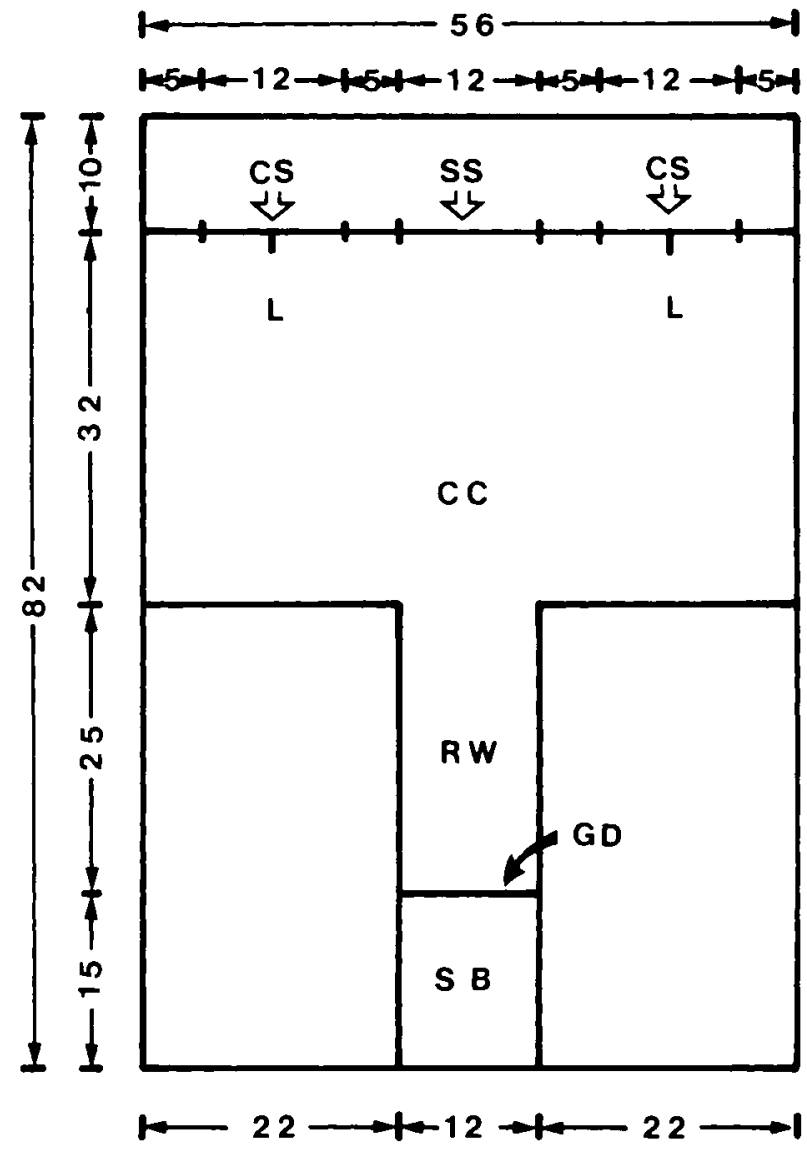

Figure 2. Diagram of the $\mathrm{T}$-maze. $\mathrm{CC}=$ choice chamber; CS = comparison stimulus; $G D=$ guillotine door; $L=$ lever; RW = runway; SB = start box; SS = sample stimulus.

two end walls and the side walls of the apparatus were mediumgray Plexiglas and the ceiling was clear Plexiglas. The startbox had a recessed food magazine in the center of one end wall and a small metal vessel, measuring $3.5 \mathrm{~cm}$ in diameter, $2 \mathrm{~cm}$ deep, and $6 \mathrm{~cm}$ wide, was sunken in the floor of the food magazine. A guillotine door opened and closed automatically to control access to the startbox. The choice chamber contained three screens, each $12 \mathrm{~cm}$ square, $10 \mathrm{~cm}$ from the floor, and $5 \mathrm{~cm}$ apart edge to edge, and two response levers, each $4 \mathrm{~cm}$ square, and $9 \mathrm{~cm}$ above the floor, at the center below both side screens. In the 2 -sec delay condition, a sample stimulus was projected automatically on the center screen as soon as an animal ran across the photobeam at the exit of the startbox. After 2 sec elapsed, the sample stimulus disappeared. After 2 more seconds elapsed, comparison stimuli were projected onto both of the side screens. The comparison stimuli disappeared when the animals pressed the correct response lever in the choice chamber, returned to the startbox, and crossed a photoelectric gate located $6 \mathrm{~cm}$ from the startbox. When the animals returned to the startbox and crossed the photoelectric gate, the guillotine door closed automatically. On the correct-response trials, the animals were given a reward within $10 \mathrm{sec}$ of returning to the startbox, and then the guillotine door opened automatically. By contrast, when the animals pressed an incorrect response lever, the comparison stimuli disappeared. When the rats returned to the startbox and crossed the photoelectric gate, the guillotine door closed automatically. That is, on the incorrect trials, the animals were retained within $10 \mathrm{sec}$ 
of returning to the startbox, and then the guillotine door opened automatically. In the 0 -sec delay condition, the comparison stimuli were turned on immediately, as the sample was turned off. Other aspects of the procedure were the same as in the 2-sec delay condition. In the simultaneous presentation condition, both the sample and the comparison stimuli were simultaneously turned on; the sample was projected onto the center screen and the comparisons were projected onto the side screens. The programming of events and data collection were carried out on line using a laboratory computer. Sound masking was provided by white noise from a blower fan.

\section{Stimuli}

A sample stimulus was rear-projected onto the center screen by means of a Chargeur Universal Kodak Ektagraphic in-line projector (Model 2). Comparison stimuli were rear-projected onto both of the side screens by means of two Handy Cabin in-line projectors (Handy Cabin Ltd). In both the 2 -sec and 0-sec delay conditions, a sample stimulus was projected for $2 \mathrm{sec}$, and comparison stimuli were projected until the animals pressed a response lever. If the response was correct, they returned to the startbox, but if the response was incorrect, the stimuli disappeared. By contrast, in the simultaneous presentation condition, both the sample and comparison stimuli were simultaneously projected until the animals pressed a response lever. Two stimuli with vertical and horizontal stripes were used, which had alternating black and white lines, $1 \mathrm{~cm}$ in width.

\section{Procedure}

Shaping of the leverpress. The animals were given leverpress training in a Skinner box $(15 \mathrm{~cm}$ in height, $22.5 \mathrm{~cm}$ in width, and $15 \mathrm{~cm}$ in length) for 5 days until they pressed the lever at least 50 times for $30 \mathrm{~min}$ a day.

Pretraining. After completing shaping of the leverpress, the animals were given pretraining for 10 days prior to the beginning of the nonmatching training until they pressed the lever in the automatic T-maze at least 30 times per day on each side. A mediumgray stimulus was projected onto the screen during both shaping and pretraining.

Nonmatching training. Of the 24 animals, 8 were trained on a 2-sec delayed nonmatching task with the vertical-horizontal-stripes stimulus set to reach a criterion in the learning for 12 trials a day (Group 2-sec delay). The next 8 animals were trained on a 0 -sec delayed nonmatching task with the vertical-horizontal-stripes stimulus set to reach the criterion for 12 trials a day (Group 0 -sec delay). The remaining animals were trained on a simultaneous nonmatching task with the vertical-horizontal-stripes stimulus set to reach the criterion for 12 trials a day (Group Simultaneous). The animals were required to choose the different comparison from a sample. In each trial, the animals started from the startbox, ran down, pressed the correct lever, and returned to the startbox. On some trials, a sample stimulus was a vertical-stripes stimulus, and on the other trials it was a horizontal-stripes stimulus, in random order in a day. The order of trials followed four predetermined random sequences. The position of a correct comparison followed four predetermined random sequences. The animals were given one 45-mg milk pellet when they made a correct response. A criterion was to achieve 11 correct trials out of a possible 12 trials a day.

\section{Results}

All the animals in Group 2-sec delay reached the learning criterion. Acquisition of nonmatching training by the 2 -sec and 0-sec delay groups were compared with acquisition of the corresponding training in Group Simultaneous. These data are summarized in Table 1 . An analysis of variance (ANOVA) using group (2-sec vs. 0-sec vs. simultaneous) was performed on the number of days
Table 1

Means $(M)$ and Standard Deviations $(S D)$ of the Number of Days to Criterion in Nonmatching Learning

\begin{tabular}{ccc}
\hline \multicolumn{1}{c}{ Group } & $M$ & $S D$ \\
\hline 2-sec delay & 60.8 & 25.2 \\
0-sec delay & 59.6 & 18.0 \\
Simultaneous & 61.1 & 25.1 \\
\hline
\end{tabular}

to criterion, revealing no significant between-group differences $(F<1)$.

Results from the early training sessions are shown in Figure 3, which shows that the percentages of correct responses differed for the three groups. That is, the analysis revealed significant between-group differences on the first $[F(2,21)=4.96, p<.05]$ and on the third session $[F(2,21)=11.77, p<.01]$. A Scheffé test was run to analyze differences in the number of correct responses on the first session among the three groups. Group 0-sec delay made significantly fewer correct responses $[F(1,21)$ $=9.79, p<.01]$, and Group 2-sec delay made marginally fewer $[F(1,21)=3.52, .05<p<.10]$ than Group Simultaneous. In addition, the percentages of correct responses on the first session were $32.3 \%$ for Group 0 -sec delay, $40.6 \%$ for Group 2-sec delay, and $53.1 \%$ for Group Simultaneous. These results suggest that Groups 0 -sec and 2-sec delay preferred the matching stimulus to the nonmatching one in the early phase of the nonmatching training, compared with Group Simultaneous.

\section{Discussion}

All the animals in Groups 2-sec and 0-sec delay learned the nonmatching task as rapidly as those in Group Simulta-

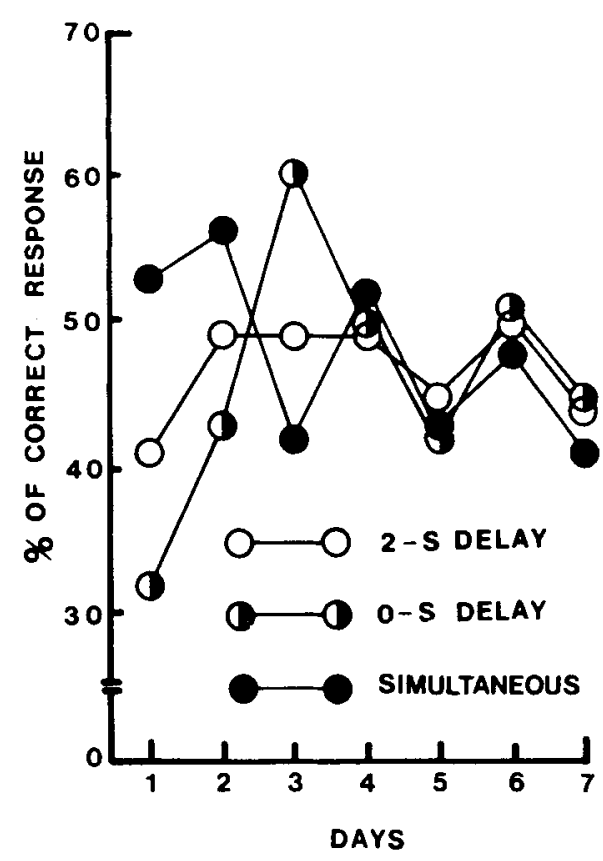

Figure 3. Performance of each group on early training sessions in nonmatching learning. 
neous. This suggests that they most likely solved the nonmatching-to-sample task by using stimuli that recurred over trials in each session in 2-sec and 0-sec delay conditions, which is not in line with Aggleton's (1985) prediction.

Performance results for the first session suggest that the rats in Groups 0-sec and 2-sec delay preferred a matching stimulus to a nonmatching one in the early phase in the nonmatching learning, compared with Group Simultaneous. This result is in line with the findings of Nakagawa (1989, 1990), in which rats learned the matching task more rapidly than the nonmatching one in simultaneous presentation situations, but it is not in line with the findings of Wilson, Mackintosh, and Boakes (1985), in which pigeons preferred the nonmatching to the matching stimulus in the matching or nonmatching task. This result also is not in line with that of Aggleton (1985), in which rats preferred the nonmatching stimulus to the matching one. The discrepancy among these experiments might be caused by both the difference in species between pigeons and rats and by the apparatus used in each experiment.

\section{EXPERIMENT 2}

The results of Experiment 1 suggest that rats can acquire delayed nonmatching learning by using stimuli that recur over trials within each session. The purpose of Experiment 2 was to examine the transfer of matching or nonmatching learning to an orthogonal dimension by using a shift-nonshift procedure (i.e., Zentall \& Hogan's 1974 design) to test the generality of formation of the relational concept that the rats acquired in the nonmatching learning in Experiment 1. The reason for this approach is that, by pitting positive transfer group (nonshifted) against negative transfer group (shifted), one may be able to detect small transfer effects more easily. If rats could learn a matching or nonmatching concept and transfer across an orthogonal dimension, then they would choose between a novel pair of stimuli in accordance with the rule that they learned in the initial learning phase-performing above chance if the rule is unchanged, and below chance if it is changed. Thus, performance on the first trial in a transfer test between Groups Nonshift and Shift would be symmetrically displaced from the chance level of performance $(50 \%)$.

\section{Method}

\section{Subjects}

Twenty-four experimentally naive male Sprague-Dawley rats were used. They were 240 days old with an initial average body weight of $545 \mathrm{~g}$. All the details of feeding schedule and handling were the same as in Experiment 1. The animals were maintained on a 7:17-h light:dark cycle, with lights off at 11:00 a.m.

\footnotetext{
Apparatus

The apparatus was basically the same as that used in Experiment 1, with the exception of both a choice chamber $(30 \mathrm{~cm}$ in height, $56 \mathrm{~cm}$ in width, and $15 \mathrm{~cm}$ in length) and a runway (30 cm in height, $12 \mathrm{~cm}$ in width, and $42 \mathrm{~cm}$ in length).
}

Stimuli

A sample stimulus was rear-projected for $2 \mathrm{sec}$ onto the center screen. After it disappeared, the apparatus was blacked out for $2 \mathrm{sec}$, and then comparison stimuli were rear-projected onto the side screens until the animals pressed a response lever. Four stimulivertical stripes, horizontal stripes, a circle with a diameter of $7.5 \mathrm{~cm}$, and an equilateral triangle with $10-\mathrm{cm}$ sides-were used. The vertical- and horizontal-stripes stimuli were the same as those used in Experiment 1.

\section{Procedure}

All the details of shaping the leverpress and pretraining were the same as in Experiment 1.

Phase 1 training. Half of the animals were trained on a delayed (2 sec) matching task with the vertical-horizontal-stripes stimulus set to reach a criterion in the Phase 1 training for 12 trials a day (Group Matching). The remaining animals were trained on a delayed $(2 \mathrm{sec})$ nonmatching task with the vertical-horizontal-stripes stimulus set to reach the criterion in the Phase 1 training for 12 trials a day (Group Nonmatching). The animals in Group Matching were required to choose the same comparison as a sample. By contrast, the animals in Group Nonmatching were required to choose the different comparison from a sample. In each trial, the animals started from the startbox, ran down, pressed the correct lever, and returned to the startbox. On some trials, a sample was a verticalstripes stimulus, and on the other trials a sample was a horizontalstripes stimulus, in random order in a day. The order of trials and the position of a correct comparison followed four predetermined random sequences. The animals were given one $45-\mathrm{mg}$ milk pellet when they made a correct response. A criterion was to achieve 11 correct trials out of a possible 12 trials in a day. All the details of Phase 1 training were the same as for Group 2-sec delay in Experiment 1 .

Phase 2 shift. After completing the Phase 1 training, half of the animals in Group Matching were trained on a matching task with a novel stimulus pair of circle-triangle (Group Nonshift: matchingmatching). The remaining animals were trained on a nonmatching task with a novel stimulus pair of circle-triangle (Group Shift: matching-nonmatching). The animals in Group Nonmatching were divided into the two subgroups of nonmatching-nonmatching (Group Nonshift) and nonmatching-matching (Group Shift) as well as in Group Matching. All the details of the Phase 2 training were the same as in the Phase 1 training.

\section{Phase 1 Training}

\section{Results}

Acquisition of the Phase 1 training by matching group was compared with acquisition of the corresponding training in Group Nonmatching. These data are summarized in Table 2. A $2 \times 2$ ANOVA was performed on the number of days to criterion, which revealed no significant main effects and no significant interaction between groups and task (all $F \mathrm{~s}<2.20$ ). Thus, there were no significant differences in the rate of learning among the four groups. The animals in Group 2-sec delay in Experiment 1 learned the nonmatching task marginally more slowly than those trained on the nonmatching task in the Phase 1 training in Experiment $2[t(18)=1.93, .05<p<.10]$.

\section{Phase 2 Shift}

Acquisition of the Phase 2 shift learning by Group Nonshift was compared with acquisition of the correspond- 
Table 2

Means $(M)$ and Standard Deviations (SD) of the Number of Days to Criterion in the Phase 1 Training

\begin{tabular}{lrr}
\hline \multicolumn{1}{c}{ Group } & \multicolumn{1}{c}{$M$} & \multicolumn{1}{c}{$S D$} \\
\hline Nonshift (Matching-Matching) & 39.7 & 7.2 \\
Shift (Matching-Nonmatching) & 39.2 & 5.5 \\
Nonshift (Nonmatching-Nonmatching) & 47.5 & 12.1 \\
Shift (Nonmatching-Matching) & 42.2 & 9.5 \\
\hline
\end{tabular}

ing shift learning in Group Shift. These data are summarized in Figure 4. An ANOVA using group (nonshift vs. shift) and task (matching vs. nonmatching) was performed on the number of days to criterion, which revealed a significant interaction between group and task $[F(1,20)=$ $4.58, p<.05]$. Group Nonshift learned the nonmatching task significantly more rapidly than did Group Shift $[F(1,20)=4.07, p<.05]$. By contrast, there were no significant between-group differences on the matching task $(F<1)$. The animals learned the nonmatching task significantly more rapidly than the matching task under the nonshift condition $[F(1,20)=6.46, p<.02]$. By contrast, there was no significant difference in the rate of shift learning between the matching and nonmatching tasks under the shift condition $(F<1)$.

In order to examine the transfer of rule learning in both matching and nonmatching tasks, performance on the first trial in the Phase 2 shift learning was analyzed. Nine animals in Group Nonshift made a correct response on the first trial in the Phase 2 shift training (75\% correct), while 3 of the animals in Group Shift made a correct response ( $25 \%$ correct). These scores have been symmetrically displaced from the chance level of performance $(50 \%)$. A chi-square test was run to analyze differences in performance on the first trial between Groups Nonshift and

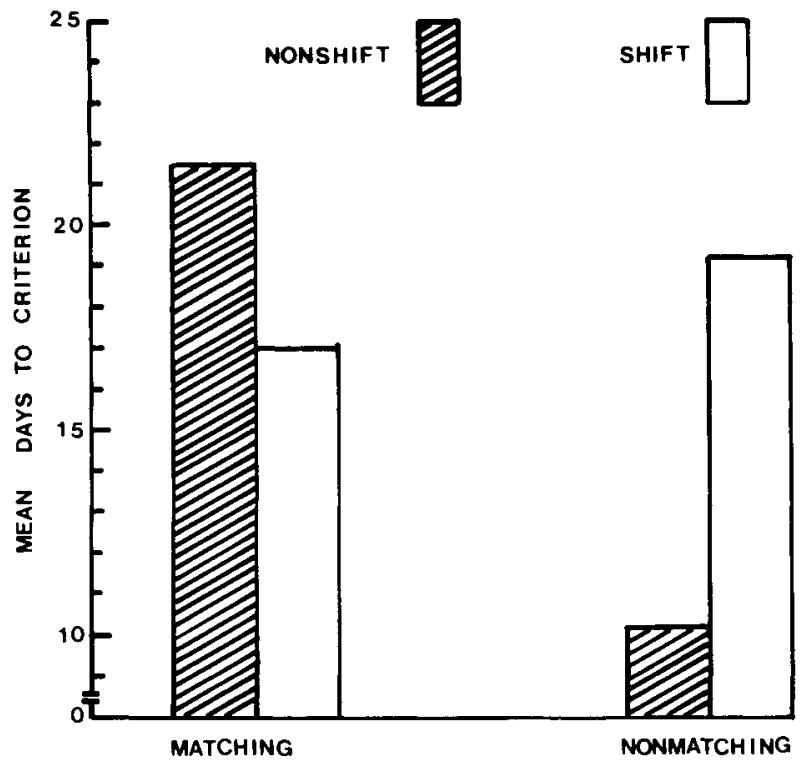

Figure 4. Mean days to criterion for each group on each task in the Phase 2 shift learning.
Shift, which yielded a significant between-group difference $\left[\chi^{2}(1)=6.00, p<.05\right]$. Four animals in Group Nonshift made a correct response on the matching task (67\% correct) and 1 animal in Group Shift made a correct response (17\% correct). On the other hand, 5 animals in Group Nonshift made a correct response on the nonmatching task (83\% correct) and 2 animals in Group Shift made a correct response (33\% correct).

\section{Discussion}

The first-trial data of the transfer test suggest that the rats chose between a novel pair of stimuli in accordance with the rule that they learned in the Phase 1 trainingperforming above chance if the rule was unchanged, below chance if it was changed. That is, performance on the first trial between Groups Nonshift and Shift have been symmetrically displaced from the chance level of performance. The days-to-criterion measure of transfer data (Figure 4) tend to agree with the first-trial data. That is, Group Nonshift (nonmatching-nonmatching) learned the nonmatching task more rapidly than Group Shift (matching-nonmatching). These results replicate those of Zentall and Hogan $(1974,1975,1976)$ with pigeons and produce several converging lines of evidence, suggesting that rats have the ability to learn the abstract concepts of matching and nonmatching.

The most interesting result was that the difference in the rate of shift learning between shifted and nonshifted rats depended on whether they were trained on matching or nonmatching. That is, the rats transferred from nonmatching to nonmatching learned more rapidly than those shifted from matching to nonmatching. By contrast, those transferred from nonmatching to matching learned more rapidly than those shifted from matching to matching. But this difference is not significant. This asymmetry of the transfer effect agrees with Nakagawa's (1992) findings using rats, and with Wilson et al. (1985) and Zentall and Hogan $(1974,1975)$ using pigeons. The pattern of this effect in the present study is the reverse of that found by Nakagawa, Wilson et al., and Zentall and Hogan, however. Thus, the cause of this asymmetry is unclear, but does not seem likely to be related to the inherent bias toward the odd stimulus that occurs both in the Phase 1 training and Phase 2 shift, as suggested by Wilson et al. But Wilson et al.'s accounts cannot readily explain the asymmetry of the transfer effect observed in the present experiment, because of the significant differences in the rate of learning between Groups Nonshift and Shift and in the rate of learning between the matching and nonmatching tasks in Group Shift.

Group 2-sec delay in Experiment 1 learned the nonmatching task marginally more slowly than did the animals trained on the nonmatching task in the Phase 1 training in Experiment 2. This discrepancy in the speed of learning might have been caused by the narrowing of the choice chamber of the apparatus - that is, narrowing the choice chamber might have reduced the animals' irrelevant response pattern such as detouring lever approach response strategy. 


\section{EXPERIMENT 3}

The results of Experiment 2 suggest that rats can acquire and transfer a relational rule (i.e., matching or nonmatching concept) across pairs of stimuli that recur over trials within each session in delayed matching or nonmatching learning. Experiment 3 was an attempt to examine transfer of matching or nonmatching learning across trial-unique stimuli using a same-different task procedure (Santiago \& Wright, 1984) to test the generality of the relational concept effect that the rats acquired using a very restricted set of repeated stimuli, as observed in Experiment 2. In this procedure, if the stimuli were identical, one special response was correct; if the stimuli were not identical, the other spe- cial response was correct. In this procedure, the animals were concurrently given training on a matching problem on some trials and a nonmatching problem on others, in a random sequence, in the same apparatus, and within each session. If the rats could learn a matching or nonmatching rule and transfer it across pairs of stimuli, then those trained on the same-different task would learn a shift task more rapidly than those trained on the other task (e.g., discrimination task) in the Phase 1 training.

\section{Method}

\section{Subjects}

Twenty-four experimentally naive male Sprague-Dawley rats were used. They were 210 days old with an initial average body weight

Table 3

The Order of Trials of a Matching and a Nonmatching Problem and the Random Presentation Sequences of Stimulus Pairs used in the Phase 1 Training and the Phase 2 Shift

\begin{tabular}{|c|c|c|c|c|c|c|c|c|c|c|c|c|c|}
\hline \multicolumn{14}{|c|}{ Random Sequence 1} \\
\hline \multicolumn{2}{|c|}{ Trial Nunber } & 1 & 2 & 3 & 4 & 5 & 6 & 7 & 8 & 9 & 10 & 11 & 12 \\
\hline \multirow{3}{*}{ Phase 1} & Group F & WW & 0000 & 吕0 & B B & BW & 므믐 & 0000 & 吅昌 & WB & 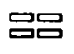 & BW & B B \\
\hline & Group F & $\Lambda \Omega$ & 00 & $\Delta O$ & $\Delta 0$ & $\Delta \Omega$ & $O \triangle$ & 00 & $O \triangle$ & $\Omega 0$ & $\Delta \Delta$ & $\Delta \Delta$ & $\Delta O$ \\
\hline & Group M & $\Delta \triangle$ & 吕品 & $\Delta O$ & $w W$ & WB & 㔛0 & $M$ & 吅 & $n_{0}$ & 00 & $\mathrm{BW}$ & $\Delta \Delta$ \\
\hline \multicolumn{2}{|c|}{ Phase 2} & ↔ & $\Delta \Delta$ & $\Delta \Omega$ & $\infty \infty \infty$ & $\infty$ & $\Delta O$ & $M$ & no & $\$ \infty \infty$ & $\infty$ & $\infty$ & $\infty \times \infty$ \\
\hline \multicolumn{14}{|c|}{ Random Sequence 2} \\
\hline \multirow{3}{*}{ Phase 1} & Group F & 唱 & B B & WB & 号象 & WW & $\mathrm{BW}$ & 吕官 & 뭄00 & WW & 品0 & WB & 0000 \\
\hline & Group $F$ & $O \triangle$ & $\Delta \Delta$ & $\Delta 0$ & $\Delta \Delta$ & $\Lambda$ & $\Delta \triangle$ & $\Delta \Delta$ & $\Delta O$ & $\Delta \Delta$ & $\Delta O$ & $\Delta 0$ & 00 \\
\hline & Group M & $O \triangle$ & 0000 & $\Delta$ & B B & $\Delta \Delta$ & WB & 吕昌 & 号00 & $\Delta \Delta$ & $\Delta \Delta$ & $\Delta \mathrm{O}$ & wW \\
\hline \multicolumn{2}{|c|}{ Phase 2} & $\Delta 0$ & $\infty \infty$ & $\$ \infty$ & $\infty$ & ţ & $\infty\}$ & $\infty$ & $\Delta \Delta$ & $f t$ & $\Delta \Delta$ & $\$ \infty$ & $\Delta$ \\
\hline \multicolumn{14}{|c|}{ Random Sequence 3} \\
\hline \multirow{3}{*}{ Phase 1} & Group F & WW & 品 & 뭄0 & BW & WB & B B & 0000 & BW & DQ00 & B B & ㅁㅁㅁㅁ & 吅 \\
\hline & Group F & $\Delta$ & $\Delta \Delta$ & $\Delta O$ & $\Delta \Delta$ & $\Delta 0$ & $\Delta \Delta$ & 00 & $\Delta \triangle$ & 00 & $\Delta \Delta$ & $O \triangle$ & $O \triangle$ \\
\hline & Group M & B B & $\Delta$ & WB & 뭄이 & $O \triangle$ & 0000 & $\Delta \Delta$ & $\Delta \triangle$ & 00 & $\Delta \Delta$ & 밈 & BW \\
\hline \multicolumn{2}{|c|}{ Phase 2} & њ & $\infty$ & $\Delta \Delta$ & $\infty \notin$ & $\$ \infty$ & $\infty \infty \infty$ & $N$ & $\infty \leftrightarrow$ & $N$ & $\infty 0 \infty$ & $\Delta 0$ & $\Delta 0$ \\
\hline \multicolumn{14}{|c|}{ Random Sequence 4} \\
\hline \multirow{3}{*}{ Phase 1} & Group $\mathrm{F}$ & 00 罗 & 0000 & 郘包 & B B & ww & WB & 㔛 & 品吕 & Ww & 吕吕 & BW & WB \\
\hline & Group F & $O \triangle$ & 00 & $\Delta O$ & $\Delta \Delta$ & $\Lambda$ & $\Delta \Delta$ & $\Delta O$ & $\Delta \Delta$ & 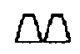 & $\Delta \Delta$ & $\Delta \Delta$ & $\Delta 0$ \\
\hline & Group M & $O \triangle$ & 0000 & $\Delta \triangle$ & 00 & B B & $\Delta \Delta$ & BW & 品吕 & WW & $\Delta \Delta$ & $\Delta 0$ & 밍 \\
\hline \multicolumn{2}{|c|}{ Phase 2} & $\Delta 0$ & $\Delta \Delta$ & $\Delta \Delta$ & $\infty \infty$ & thes & $\oiint \infty$ & $\Delta \Delta$ & $\infty$ & $t x^{3}$ & $\infty$ & $\infty$ & $\$ \infty$ \\
\hline
\end{tabular}

Notes: $g=$ horizontally striped; $\mathbf{D}=$ vertically striped; $\mathbf{B}=$ black; $\mathbf{W}=$ white. 
of $370 \mathrm{~g}$. The feeding schedules and handling were the same as in Experiment 1. The animals were maintained on a 7:17-h light:dark cycle, with lights off at 11:00 a.m.

\section{Apparatus}

The apparatus was the same as that used in Experiment 1.

\section{Stimuli}

The stimuli were rear-projected onto the center screen and medium-gray stimuli were rear-projected onto both of the side screens. The stimuli used were as follows: an equilateral triangle with $4.1-\mathrm{cm}$ sides; a circle with a diameter of $3.2 \mathrm{~cm}$; a large isosceles trapezium with a $3.0-\mathrm{cm}$ upper base, a $5.2-\mathrm{cm}$ lower base, and a height of $3.0 \mathrm{~cm}$; a small isosceles trapezium with a $1.4-\mathrm{cm}$ upper base, a $3.2-\mathrm{cm}$ lower base, and a height of $1.8 \mathrm{~cm}$; a diamond with $4.5-\mathrm{cm}$ long diagonal lines and $4.2-\mathrm{cm}$ short diagonal lines; a $7.36 \mathrm{~cm}^{2}$ cross; a double circle, $5.4 \mathrm{~cm}$ in diameter; a white card with $5.8-\mathrm{cm}$ squares; and a black card with $5.8-\mathrm{cm}$ squares; vertical stripes; and horizontal stripes. The vertical- and horizontalstripes stimuli had alternating black and white lines, $0.45 \mathrm{~cm}$ in width. An identical and a different stimulus consisted of 2 out of 11 stimulus items, as shown in Table 3. A sample of an identical and a different stimulus are illustrated in Figure 5. For a successive discrimination task, the vertical- and horizontal-stripes stimuli were used, which had alternating black and white lines, $1 \mathrm{~cm}$ in width. The size of these stimuli for the discrimination task was $12 \mathrm{~cm}$ squares.

\section{Procedure}

The shaping of the leverpress, handling, and pretraining were the same as in Experiment 1.

Phase 1 training. Sixteen of the animals were trained on a samedifferent task to reach a criterion in the Phase 1 training for 12 trials a day, in which they were given a matching problem with an identical stimulus on some trials and a nonmatching problem with a different stimulus on the other trials, in a random order within each session. On a matching trial, the animals were required to press the right lever, and on a nonmatching trial they were required to press the left lever. The order of trials followed four predetermined random sequences (see Table 3). Half of these animals were trained on 8 same-different tasks for 12 trials a day (Group F), and the remaining animals were trained on 16 same-different tasks for 12 trials a day (Group M), in which trial-unique (nonrecurring) stimuli were used. The animals in Group $F$ were trained on the same-different task consisting of the large isosceles trapezium, the small isosceles trapezium, or the circle and triangle items, or on the same-different task consisting of the white, black, vertical-stripes and horizontal-stripes items. The animals in Group $M$ were trained on the same-different task consisting of the triangle, the circle, black, white, the large isosceles trapezium, the small isosceles trapezium, and the vertical-and horizontal-stripes items. The identical and different stimuli, and the order of trials of a matching and a nonmatching problem used in both the Phase 1 training and the Phase 2 shift are shown in Table 3.

The last 8 animals were trained on a successive discrimination task (vertical-horizontal-stripes task) with a noncorrection training method (Group Control: C). On the vertical-stripes stimulus trials, the animals were required to press the right lever, and were required to press the left lever on the horizontal-stripes stimulus trials. The order of trials followed four predetermined random sequences.

The animals were given one 45 -mg milk pellet when they made a correct response. A criterion was to achieve 11 correct trials out of a possible 12 trials per day in every group.

Phase 2 shift. After completing the Phase 1 training, all the animals were trained on a new same-different task consisting of cross, double circle, diamond, and large isosceles trapezium items to a criterion in the Phase 2 shift for 12 trials in a day. Only the identical stimulus consisting of two large isosceles trapezium items was used in both the Phase 1 training and the Phase 2 shift. All the details of training in the Phase 2 shift were the same as in the Phase 1 training for Groups $F$ and $M$.

\section{Results}

\section{Phase 1 Training}

Acquisition of the Phase 1 training by the groups trained on the same-different task was compared with acquisition of the corresponding training in Group $\mathrm{C}$. These data are summarized in Figure 6. An ANOVA using group (C vs. F vs. M) was performed on the number of days to criterion, which revealed no significant between-group differences $[F(2,21)=1.34]$.

\section{Phase 2 Shift}

Acquisition of the Phase 2 shift learning by Groups $F$ and $M$ was compared with acquisition of the corresponding shift learning in Group $\mathrm{C}$. These data are summarized

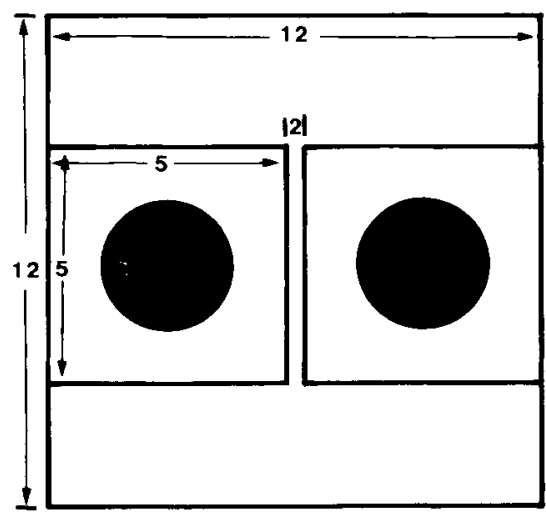

IDENTICAL STIMULUS

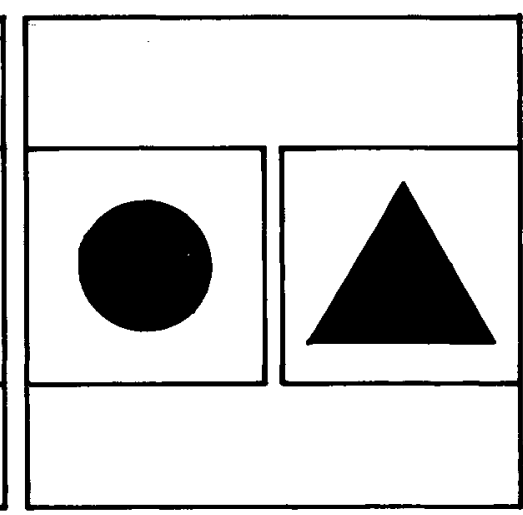

DIFFERENT STIMULUS

Figure 5. A sample of both the identical and different stimuli used in Experiment 3, with measurements in centimeters. 


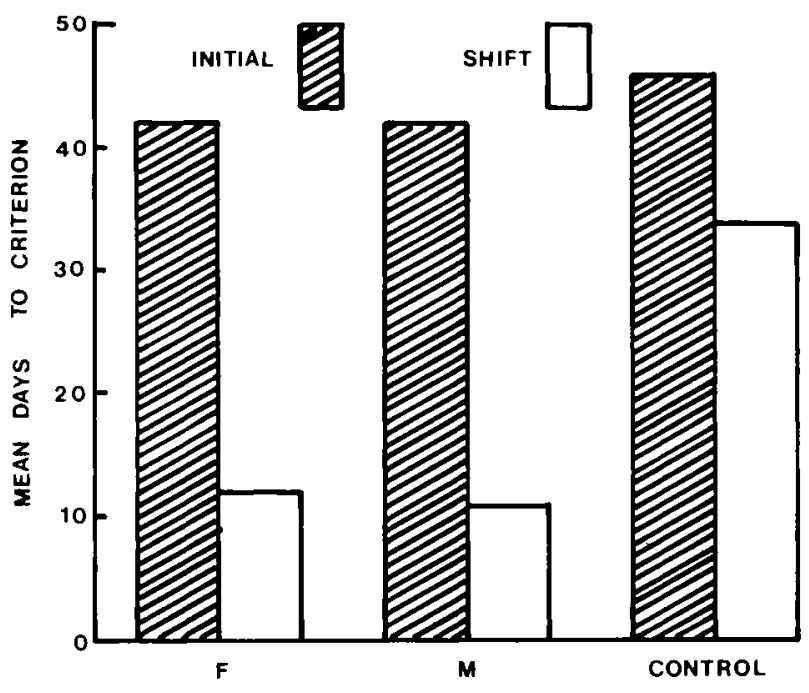

Figure 6. Mean days to criterion for each group in both the initial and shift learnings.

in Figure 6. An ANOVA using group (C vs. F vs. M) was performed on the number of days to criterion, which revealed significant between-group differences $[F(2,21)$ $=14.20, p<.01]$. A Scheffé test was run to analyze differences in the number of days to criterion among the three groups, and showed that Group $\mathrm{F}[F(1,21)=23.06$, $p<.001]$ and Group $\mathrm{M}[F(1,21)=25.54, p<.001]$ learned significantly more rapidly than Group $\mathrm{C}$ did. But there was no significant difference in the number of days to criterion between Groups $\mathrm{F}$ and $\mathrm{M}(F<1)$.

\section{Discussion}

The days-to-criterion measure of transfer data makes it clear that the rats performed on novel stimuli in accordance with the rule that they acquired in the Phase 1 training. This result agrees with the findings of Aggleton (1985), and indicates that rats can acquire and transfer matching and nonmatching rules across trial-unique stimuli. That is, these results replicate the relational concept effect.

\section{GENERAL DISCUSSION}

In three experiments, it was examined whether rats have an ability to form a relational rule. In Experiment 1, they were trained on a nonmatching task under a 2-sec delay, a 0 -sec delay, or a simultaneous condition. All the animals in Groups 2-sec and 0-sec delay learned the nonmatching task as rapidly as Group Simultaneous did. In Experiment 2, the rats were trained on a delayed matching or nonmatching task and then under either shift or nonshift conditions by using stimuli that recurred over trials within each session. Group Nonshift made significantly more correct responses on the first trials in Phase 2 than Group Shift did. Group Nonshift learned the nonmatching task significantly more rapidly than Group Shift did. These results are in accordance with the findings of Nakagawa (1992). In Experiment 3, the rats were trained on a same-different task and then shifted to novel same-different tasks, where Groups $\mathrm{F}$ and $\mathrm{M}$ learned the shift task significantly more rapidly than Group $\mathrm{C}$ did. This result is in accordance with the findings of Aggleton (1985), Mumby et al. (1990), and Rothblat and Hayes (1987). Thus, the results of the present three experiments produce several converging lines of evidence suggesting that rats have the ability to learn the abstract concepts of matching-nonmatching and same-different.

The main purpose of the present studies was to confirm that rats can acquire and transfer a relational concept across an orthogonal dimension. In Experiment 1, it was found that they learned a nonmatching rule under the 2-sec delay between sample-offset and comparisononset conditions. In Experiments 2 and 3, it was found that they acquired and transferred a matching or nonmatching rule to novel pairs of stimuli-a very restricted set of repeated stimuli as well as trial-unique stimuli. Thus, it is reasonable to assume that the results of these three experiments suggest that rats have the ability to learn a relational concept.

\section{REFERENCES}

AGgLeton, J. P. (1985). One-trial object recognition by rats. Quarterly Journal of Experimental Psychology, 37B, 279-294.

LASHLEY, K. S. (1938a). Conditional reaction in the rats. Journal of Psychology, 6, 311-324.

LASHLEY, K. S. (1938b). The mechanism of vision: XV. Preliminary studies of rats' capacity for detail vision. Journal of Genetic Psychology, 18, 176-177.

Lombardi, C. M., Fachinelli, C. C., \& Delius, J. D. (1984). Oddity of visual patterns conceptualized by pigeons. Animal Leaming \& Behavior, 12, 2-6.

Mumby, D. G., Pinel, J. P., Wood, E. R. (1990). Nonrecurringitems delayed nonmatching-to-sample in rats: A new paradigm for testing nonspatial working memory. Psychobiology, 18, 321-326.

NAKAGAWA, E. (1989). Matching and oddity learnings in white rats. Memoirs of the Faculty of Education, Kagawa University: II, 39, 29-38.

NAKAGAWA, E. (1990). Transfer of rule learning in rats. Memoirs of the Faculty of Education, Kagawa University: I, 80, 89-104.

NAKAGAWA, E. (1992). Transfer of matching and nonmatching concept in rats. Psychobiology, 20, 311-314.

Rothblat, L. A., Hayes, L. L. (1987). Short-term object recognition memory in the rat: Nonmatching with trial-unique junk stimuli, Behavioral Neuroscience, 101, 578-590.

Santiago, H., WRight, A. A. (1984). Pigeon memory: Same/different concept learning, serial probe recognition acquisition, and probe delay effects on the serial-position function. Journal of Experimental Psychology: Animal Behavior Processes, 10, 498-512.

URCUIOLI, P. J. (1977). Transfer of oddity-from-sample performance in pigeons. Journal of the Experimental Analysis of Behavior, 27, 195-202.

UrCuiol, P. J., \& Nevin, J. A. (1975). Transfer of hue matching in pigeons. Journal of the Experimental Analysis of Behavior, 24, 149-155.

Wilson, B., Mackintosh, N. J., \& Boakes, R. A. (1985). Matching and oddity learning in the pigeon: Transfer effects and the absence 
of relational learning. Quarterly Joumal of Experimental Psychology, 37B, 295-311.

Wodinsky, J., \& Bitrerman, M. E. (1953). The solution of oddity problems by the rat. American Journal of Psychology, 66, 137-140.

ZeNTALl, J. R., \& Hogan, D. E. (1974). Abstract concept learning in the pigeon. Journal of Experimental Psychology, 102, 393-398.

Zentall, J. R., \& Hogan, D. E. (1975). Concept learning in the pigeon: Transfer to new matching and nonmatching stimuli. American Journal of Psychology, 88, 233-244.

Zentall, J. R., \& Hogan, D. E. (1976). Pigeons can learn identity or difference. Science, 191, 408-409.

(Manuscript received July 24, 1992; revision accepted for publication March 4, 1993.) 